\title{
THE CAPTURE AND 3-D CULTURE OF VIABLE CIRCULATING TUMOR CELLS USING HIGH OPEN-FACTOR PARYLENE-C/HT MEMBRANE FILTERS
}

\author{
B. $L u^{I^{*}}, T . X u^{2}, A$. Goldkorn ${ }^{2}$, and Y. C. Tai ${ }^{1}$ \\ ${ }^{1}$ California Institute of Technology, Pasadena, California, USA \\ ${ }^{2}$ University of Southern California, Los Angeles, California, USA
}

\begin{abstract}
This paper presents an improved method of capturing viable circulating tumor cells (CTC) from human blood using low-constant-pressure filtration through a high open-factor parylene-C membrane filter with parylene-HT surface coating. More than $90 \%$ viable CTCs can be captured on the filter, with minimal blood cells left. For the first time, we demonstrated the feasibility of on-filter 3D culture (with significant cell proliferation) from captured cancer cells. This work is one-step closer to the final goal of capturing and culturing CTCs directly from cancer patient blood to enable cancer analysis and management.
\end{abstract}

\section{INTRODUCTION}

Cancer metastasis is still the main cause of death for patients having solid-tumor cancers. Circulating tumor cells (CTC) are tumor cells disseminated from the primary tumor into blood stream. The presence of CTC in peripheral blood has important clinical significance for patients with various types of cancers. Study of CTC will also provide valuable insight into the mechanism of tumor metastasis.

Most current CTC assays are used either for the enumeration of CTCs by immunostaining [1-5], or for specific molecular analysis by quantitative PCR (qPCR) [2,3]. For both applications, the main challenge is the extremely low concentration of CTC (e.g. $\sim 1 / \mathrm{mL}$ ) in the patient blood. Therefore, an enrichment step of rare CTCs from a large volume (e.g. in the milliliter range) of patient blood sample is crucial for successful CTC study. So far, the reported CTC enrichment techniques include the density gradient centrifugation [3], immunomagnetic separation [3], microfluidic devices combined with immunoaffinity based selection $[4,5]$ and size based filtration [1-3]. Immunoaffinity based methods capture CTCs by using cancer specific markers [3-5]. Non-cancer blood cells can be efficiently removed, resulting in high CTC enrichment. However, the CTC capture efficiency greatly depends on the biomarkers selected, and the throughput is usually low because of the incubation time required. Size based filtration has been explored for solid-tumor cancers because the herein epithelial CTCs (15-30 $\mu \mathrm{m}$ in diameter) are generally larger and less deformable than normal blood cells [1-3]. Among various filter designs, membrane filters have the advantage of short operation time (i.e. high throughput). Previously, we reported parylene-C based dual-layer [1] and single-layer [2] membrane filters, which could capture viable CTCs with high capture efficiency and viability. However, the CTC enrichment was not good enough because too many blood cells were also captured after filtration.

Further analyses of CTCs after capture can enable the characterization of the original tumor and greatly benefit future genetic and chemotherapy studies. However, these studies are usually restricted by the rarity of available CTCs after capture. Therefore, a CTC culture step after capture is highly desirable. Current CTC culture methods focus on conventional 2D culture, where cells are cultured as a monolayer on a substrate [2,5]. Recently, researchers are recognizing the limitations of 2D culture, given that it cannot fully reproduce the in vivo cellular microenvironment with cell-cell and cell-matrix interactions [6,7]. To better recapitulate the morphology and features the in vivo cells have, 3D culture models, which provide more in vivo-like conditions and realize the original growth characteristics of the tumors, are attracting increasingly more attentions [6,7].

In this work, we present the use of a netlike high open-factor (i.e., area of openings/total area) parylene-C slot filter (coated with parylene-HT) with a low-constant-pressure filtration system to capture viable CTCs from blood. More than $90 \%$ viable cancer cells were consistently recovered from whole blood, with minimal blood cells remaining on filter. The CTC enrichment was 1 and 2 order(s) of magnitude higher than our previously reported single-layer [2] and dual-layer filters [1], respectively. More excitingly, for the first time, we demonstrated the feasibility of on-filter 3D culture (with significant cell proliferation) from captured cancer cells.

\section{EXPERIMENTAL}

\section{Filter Design and Fabrication}

Parylene-C was chosen as the filter material because of its unique properties [1]. It is mechanically strong (Young's modulus $\sim 4 \mathrm{GPa}$, and tensile strength $\sim 70 \mathrm{MPa}$ ) while malleable (elongation to break $\sim 200 \%$ ). It is optically transparent in the visible range, which facilitates direct pathological observation of stained cells under microscope. It is inert to most of the chemicals and solvents used in standard chemical and biological laboratories. Moreover, its biocompatibility makes parylene-C based devices suitable for cell culture applications. Finally, high quality pin-hole free parylene-C thin film can be obtained with room-temperature chemical vapor deposition (CVD), and further patterned with lithography and oxygen plasma by reactive ion etching (RIE).

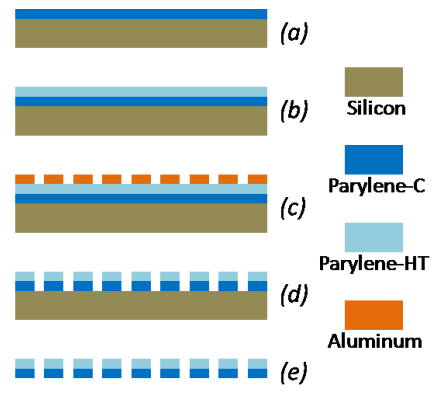

Figure 1: Filter fabrication process. (a) $9 \mu m$ parylene-C deposition; (b) $0.5 \mu \mathrm{m}$ parylene-HT deposition; (c) Aluminum deposition and wet-etching patterning; (d) Pattern parylene layers by RIE, with Al mask; (e) Release parylene filter from Si substrate.

Parylene-C and parylene-HT dimers were purchased from Specialty Coating Systems. The fabrication process started with a $9 \mu \mathrm{m}$ parylene-C film deposition on silicon wafer (Fig. 1). A $0.5 \mu \mathrm{m}$ parylene-HT (chemical structure shown in Fig. 2) was then coated on parylene-C surface. This inert and hydrophobic parylene-HT coating could efficiently prevent cancer cell adhesion on the filter surface, which was important for 3D tumor culture application. Aluminum was deposited using a thermal evaporator, followed by lithography and wet-etching patterning. For each filter, slot arrays were etched through the parylene-C film by RIE in a $6 \mathrm{~mm} \times 6 \mathrm{~mm}$ 
square region. Each slot size was $6 \mu \mathrm{m} \times 30 \mu \mathrm{m}$, which was optimal according to our previous publication [2]. The patterned parylene membrane was peeled off from the silicon substrate. The fabricated filter looked like a net, with a high open-factor of $42 \%$ (Fig. 3).

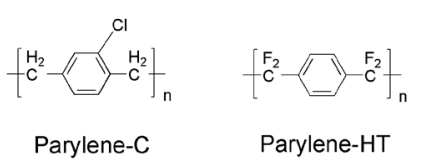

Figure 2: Chemical structures of parylene-C and parylene-HT film.

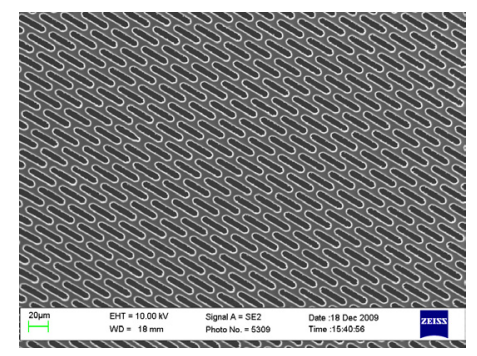

Figure 3: SEM picture of the fabricated membrane filter.

\section{Filtration Procedure}

Four pieces of PDMS sheets were used to sandwich the parylene filter, and form a top and bottom chambers (Fig. 4(b)). The top chamber had the dimension of $6 \mathrm{~mm} \times 6 \mathrm{~mm} \times 1.5 \mathrm{~mm}$. Two acrylic sheets with central holes were also used. The assembly was clamped to ensure good sealing and prevent leakage.

The CTC filtration was carried out with a low-constant-pressure fluid delivery system (Fig. 4(a)), which was connected to the filter assembly. During filtration, sample was driven into the filter assembly by $\mathrm{N}_{2}$ with low drive pressure P. Low drive pressure ensured a low pressure drop $\Delta \mathrm{P}$ across the filter, which was crucial for the high viability of captured CTCs. In previous filtration approaches, sample was driven by direct hand-push or syringe pump [1,3]. Although a near-constant flow rate could be achieved, $\Delta \mathrm{P}$ increased when the filter was gradually and partially clogged. In contrast, the low-constant-pressure fluid delivery system could keep a near-constant low $\Delta \mathrm{P}$ during filtration.

We used non-small-cell prostate cancer PC-3 cells for device testing. PC-3 cell line was cultured in RPMI 1640 culture medium (Mediatech, Inc.) supplemented with $10 \%$ fetal bovine serum (RPMI complete medium) at $5 \% \mathrm{CO}_{2}$ and $37^{\circ} \mathrm{C}$. PC-3 cells were pre-labeled with calcein-AM fluorescent dye (Invitrogen), centrifuged and then re-suspended in phosphate buffered saline (PBS). A model system was constructed by spiking PC-3 cells at 100 cells $\mathrm{mL}^{-1}$ into $1 \mathrm{~mL}$ human whole blood obtained from a healthy donor. We used $0.1 \mathrm{psi}$ low pressure to drive the sample during filtration. Filtration of $1 \mathrm{~mL}$ whole blood took about $5 \mathrm{~min}$. After filtration, capture efficiency (Eq. (1)) was measured by counting the captured cancer cells under epifluorescence microscope. The viability (Eq. (2)) of captured cancer cells was evaluated by on-filter staining with Propidium Iodide (PI) (Invitrogen). Enrichment (Eq. (3)) was determined by staining white blood cells (WBCs) remaining on filter with the nucleus dye Acridine Orange (AO) (Invitrogen) followed by WBC counting.

$$
\begin{gathered}
\text { Capture Efficiency }=\frac{\# \text { of captured cancer cells }}{\# \text { of spiked cancer cells }} \\
\text { Viability }=\frac{\# \text { of viable cancer cells }}{\# \text { of captured cancer cells }}
\end{gathered}
$$

$$
\begin{aligned}
& \text { CTC enrichment }(\text { fold })= \\
& \frac{[\# \text { cancer cells } / \# W B C s] \text { on filter after filtration }}{[\# \text { cancer cells } / \# W B C s] \text { in original sample }}
\end{aligned}
$$

\section{Cell-substrate adhesion assay}

To examine cancer cell adhesion on parylene- $\mathrm{C}$ with different surface conditions, four types of surfaces were used in this study, including untreated parylene- $\mathrm{C}$ film, $\mathrm{O}_{2}$ plasma treated parylene- $\mathrm{C}$ film, parylene-C film with parylene-HT coating and polystyrene substrate. Plasma treated parylene-C sample was prepared by treating parylene-C film in a plasma etching machine for $1 \mathrm{~min}$ (100W, 200mtorr). Parylene-HT coating was obtained by depositing $0.5 \mu \mathrm{m}$ parylene-HT on parylene-C film. Polystyrene substrate is widely used in cell culture and was used here as a control for cell adhesion assay. Each substrate was sterilized in $70 \%$ ethanol and PBS before experiments. Contact angle measurements were performed by measuring the contact angles of deionized water drops on different substrates by using a NRL Contact Angle Goniometer (Ramé-Hart instrument co.). To determine the cell adhesion, PC-3 cells were counted and loaded onto each substrate. After 10 hours of incubation, the substrates were washed twice with PBS. Cells remaining on the substrates were then counted to determine the percentage of adhering cells.

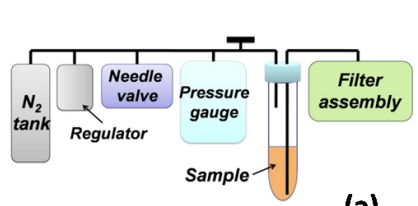

(a)

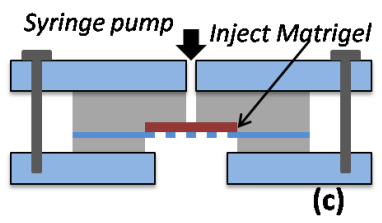

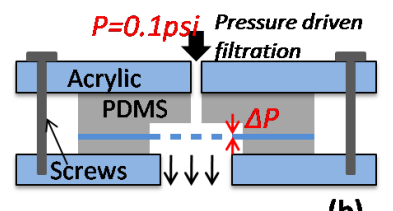

(b)

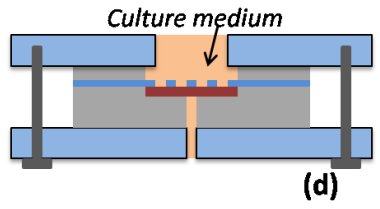

Figure 4: Operation procedure. (a) Schematic of the low-constant-pressure filtration system; (b) Filter assembly and filtration process; (c) Matrigel injection after filtration; (d) Schematic of $3 D$ on-filter tumor culture.

\section{D tumor culture}

To demonstrate the feasibility of on-filter 3D culture of the captured cancer cells after filtration, we spiked about 30 unlabeled PC-3 cancer cells into $1 \mathrm{~mL}$ PBS. The sample was filtrated and cancer cells were captured on the filter. Matrigel was selected as the 3D scaffold material. A 1:1 mixture of Matrigel (BD Biosciences) and RPMI complete medium was carefully injected into the filter top chamber by a syringe pump, to prevent the cell damage and undesirable bubble appearance. Gelation was allowed to occur at $37^{\circ} \mathrm{C}$ for $30 \mathrm{~min}$. Then the filter assembly was placed invertedly and covered with RPMI complete medium. The whole setup was left in a petri-dish and placed in an incubator. Culture medium was refreshed every day. Calcein-AM and PI live/dead cell staining was used again to determine the cellular viability of the 3D tumor.

2D monolayer cancer cell culture without Matrigel injection was used as a control. For 2D on-filter culture, membrane filter was disassembled after filtration and soaked in the culture medium.

\section{Immunofluorescence and image acquisition}

Immunofluorescence staining was performed on the 3D tumor after 8 days of culture. FITC-conjugated anti-EpCAM antibody 
(BioLegend) and PE-conjugated anti-CD49b antibody (BD Biosciences) were used to immunostain the cancer cell membranes. After staining, images were taken by a Nikon E800 epifluorescence microscope (Nikon Inc.), equipped with a CCD camera.

\section{SIMULATION}

In order to estimate the shear stress and tension exerted on the captured cancer cells during filtration, we used finite-element simulation tool COMSOL Multiphysics. 2D incompressible Navier-Stokes steady-state analysis model was selected. The dynamic viscosity of blood was set as $0.003 \mathrm{~Pa} \cdot \mathrm{s}$. Drive pressure $\mathrm{P}=$ 0.1 psi. Simulated 2D flow velocity profile in the filter chambers was shown in Fig. 5. A nearly uniform flow field was achieved in the filter region. Based on this velocity profile, we calculated the maximum shear stress and surface tension on the cancer cells captured at different locations (Fig. 6). Due to the low trans-filter pressure drop $\Delta \mathrm{P}$, the shear stress and tension were also low, ensuring the high viability of the cancer cells [8].

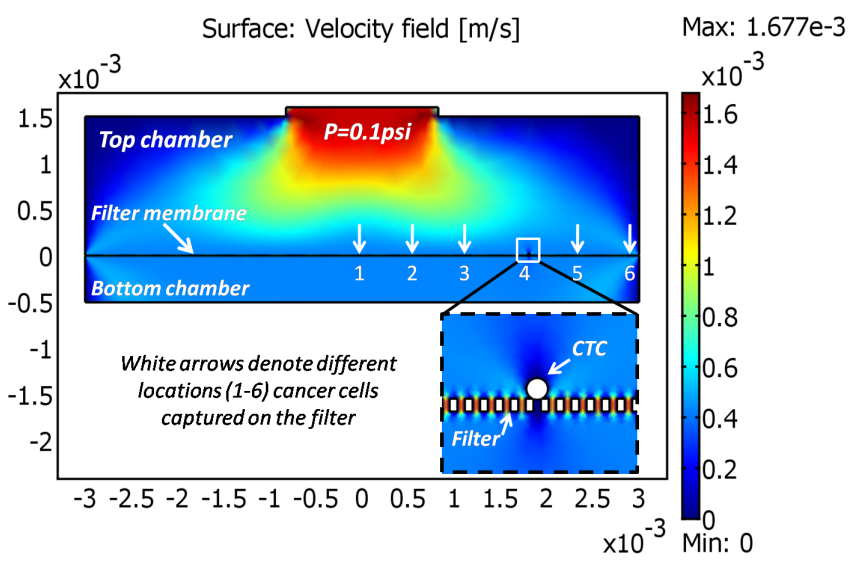

Figure 5: FEM simulation result of the flow velocity field during filtration. The inset shows an example of captured CTC.

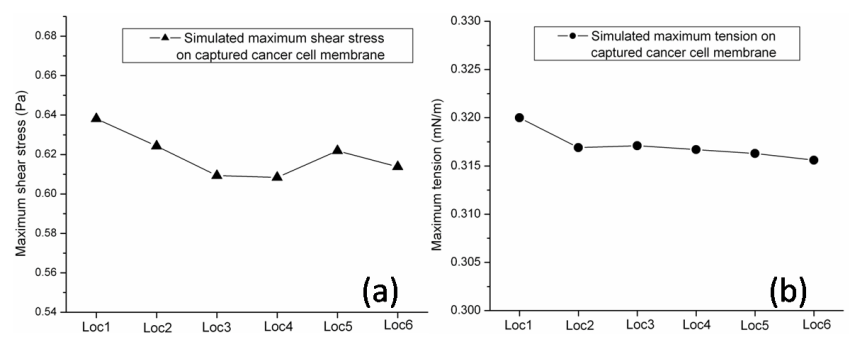

Figure 6: Simulated maximum shear stress (a) and tension (b) on CTC membrane captured at different locations (Location 1-6 were labeled in Fig. 5).

\section{RESULTS AND DISCUSSION}

The large open-factor contributed in two aspects to the improved filter performance. Firstly, the large open-factor decreased the flow resistance during filtration. Reduced flow resistance lowered the drive pressure required for successful sample processing and the trans-filter pressure drop $\Delta \mathrm{P}$, which ensured the high viability of captured cancer cells. Filter testing with the model system showed that the capture efficiency was $92.4 \%(\mathrm{SD}=7.4 \%)$, and the viability was measured to be $93.8 \%$ ( $\mathrm{SD}=3.4 \%$ ). Fig. 7 shows the examples of live and dead cancer cells using calcein-AM and PI live/dead staining assay.
Secondly, the large open-factor also decreased the filter surface area. Because of their smaller size and larger deformability, most blood cells can deform and pass through the slots during filtration. However, some blood cells may remain on the filter top surface, resulting in a non-ideal CTC enrichment. Reduced filter surface area greatly lowers the chance of blood cell retention on filter surface, thus improving the enrichment. Compared to the $\sim 200$-fold enrichment of our previously reported filter (open-factor $18 \%$ ) [2], this new filter (open-factor $42 \%$ ) achieved a 2149 -fold $(\mathrm{SD}=1120)$ enrichment. Fig. $7(\mathrm{c})$ shows that there were still minimal amount of blood cells remaining on the filter, possibly due to the friction between the cell and slot side walls.

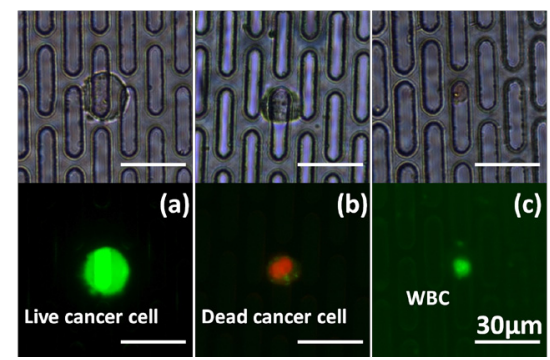

Figure 7: Live/dead staining assay of captured cancer cells. (a) live cancer cell retained calcein-AM; (b) dead cancer cell was stained with PI; (c) later on, remaining WBC was stained with the nucleus dye AO. (scale bars: $30 \mu \mathrm{m})$

For on-chip 3D cell culture, cell-cell and cell-extracellular matrix (ECM) interactions should dominate over cell-substrate interaction. Therefore, we needed to treat the filter surface to be resistant to cancer cell adhesion. Surface property and cancer cell adhesion evaluations of untreated parylene- $\mathrm{C}, \mathrm{O}_{2}$ plasma treated parylene- $\mathrm{C}$, parylene- $\mathrm{C}$ with parylene-HT coating, and polystyrene are shown in Fig. 8(a) \& (b). Untreated parylene-C surface was hydrophobic. While plasma treatment could turn it to be hydrophilic, parylene-HT coating rendered the surface to be even more hydrophobic. After 10 hours of incubation, about 30\% PC-3 cancer cells remained adhering on the untreated parylene-C surface. Although untreated parylene-C surface could be considered as cell resistant compared to the tissue culture polystyrene control, it was still not suitable for 3D tumor culture. Fig. 8(c) shows that during 3D tumor culture, some cancer cells adhered and proliferated two-dimensionally on the untreated parylene-C filter, which was undesirable. It was noticed that plasma treated parylene-C surface displayed enhanced adhesion. In comparison, parylene-HT coated surface heavily repelled cancer cell adhesion, reducing the cell adhesiveness to be only one-third of the original level.

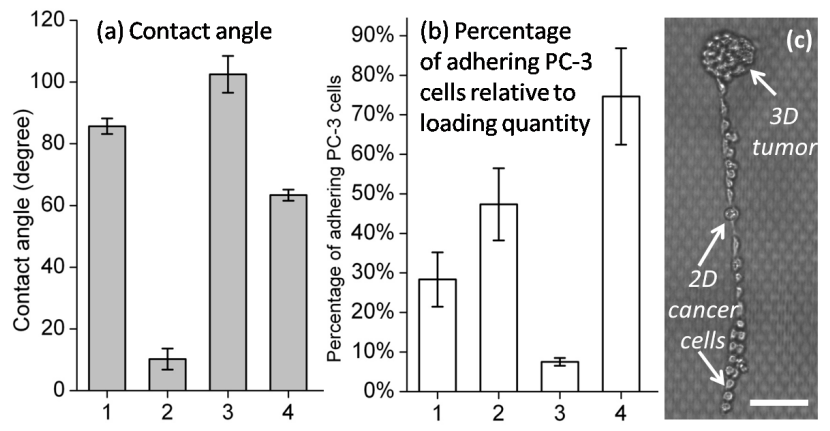

Figure 8: Parylene surface treatment. 1: untreated parylene-C surface; 2: $\mathrm{O}_{2}$ plasma treated parylene-C; 3: parylene-C with 
parylene-HT coating; 4: tissue culture polystyrene substrate. (a) contact angle measurement; (b) evaluation of cancer cell adhesiveness; (c) without parylene-HT coating, some cancer cells adhered and proliferated on untreated parylene- $C$ filter surface during 3D tumor culture with Matrigel. (scale bar: 100 $\mu \mathrm{m}$ )

Therefore, parylene-HT coated parylene-C filter was selected for 3D culture after filtration. Captured PC-3 cancer cells proliferated three-dimensionally into the Matrigel, and gradually formed a spherical tumor. Matrigel is a biomatrix hydrogel containing many essential components of the ECM such as collagen, laminin, entactin and other important growth factors, which can support cellular proliferation and induce cellular differentiation $[6,7]$. The cellular proliferation rate was monitored in Fig. 9(a). On the $6^{\text {th }}$ day, live/dead staining was used to evaluate the cellular viability inside the tumor (Fig. 9(b)-(e)). Over 90\% cells kept viable during the tumor formation. To further investigate the structure of the tumor, after 8 days of culture, we stained the cell membranes with cancer specific immunofluorescence surface markers (Fig. 10). The images indicated that separate cells were adhering to form a combined structure. Cell-cell and cell-ECM interactions were clearly observed. In comparison, 2D on-filter culture was used as a control (Fig. 10). Cells attached to parylene-C filter, and formed a confluent 2D monolayer after 8 days of culture.

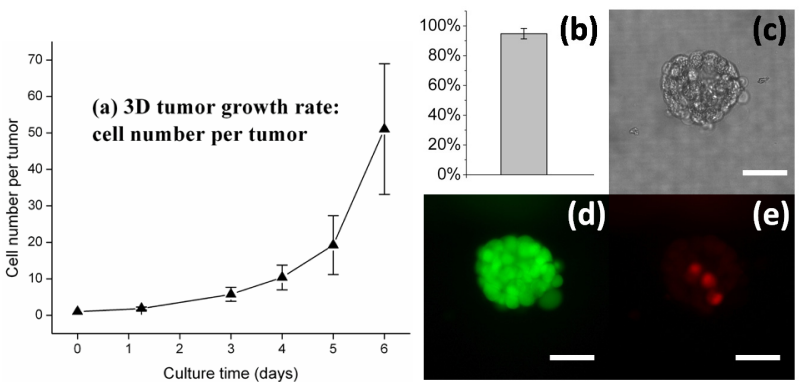

Figure 9: 3D on-filter cultured tumor growth rate and viability. (a) growth rate; (b) viability on the $6^{\text {th }}$ day; (c)-(e) bright field and fluorescence images of calcein-AM \& PI stained tumor. (all scale bars: $40 \mu \mathrm{m}$ )

\section{CONCLUSION}

In this paper we report the use of a high open-factor parylene-C/HT filter for the capture of CTCs from whole blood, with high capture efficiency, viability, enrichment and throughput. Due to the large open-factor, CTC enrichment was much higher than our previous filters. Moreover, for the first time, we demonstrated the feasibility of 3D tumor culture after filtration. Captured cancer cells were cultured on-filter, and proliferated into Matrigel three-dimensionally. Live/dead staining assay and immunofluorescence staining verified the cellular viability and the morphology of the 3D tumor. Our ongoing work includes the capture and culture of CTCs directly from clinical patient blood sample, which can enable the characterization of the original tumor and greatly benefits the future genetic and chemotherapy studies.

\section{ACKNOWLEDGEMENT}

This work is supported by the Caltech CI2 I-Grant Award.

\section{REFERENCES}

[1] S. Zheng, H. Lin, R. J. Cote and Y. -C. Tai, "A novel 3D micro membrane filtration device for capture viable rare circulating tumor cells from whole blood", Proc. of Hilton Head 2008, Hilton Head Island, SC, USA, 2008, pp. 134-137.

[2] B. Lu, T. Xu, S. Zheng, A. Goldkorn and Y. -C. Tai, "Parylene membrane slot filter for the capture, analysis and culture of viable circulating tumor cells", Proc. of MEMS 2010, Hong Kong, China, 2010, pp. 935-938.

[3] P. Paterlini-Brechot and N. L. Benali, "Circulating tumor cells (CTC) detection: Clinical impact and future directions", Cancer Letters, vol. 253, pp. 180-204, 2007.

[4] S. Nagrath, L. V. Sequist, S. Maheswaran, et al. "Isolation of rare circulating tumor cells in cancer patients by microchip technology", Nature, vol. 450, pp. 1235-1239, 2007.

[5] K. T. Helzer, H. E. Barnes, L. Day, et al. "Circulating tumor cells are transcriptionally similar to the primary tumor in a murine prostate model", Cancer Research, vol. 69, pp. 7860-7866, 2009.

[6] Y. Torisawa, H. Shiku, T. Yasukawa, et al. "Multi-channel 3-D cell culture device integrated on a silicon chip for anticancer drug sensitivity test", Biomaterials, vol. 26, pp. 2165-2172, 2005.

[7] T. R. Sodunke, K. K. Turner, S. A. Caldwell, et al. "Micropatterns of Matrigel for three-dimensional epithelial cultures", Biomaterials, vol. 28, pp. 4006-4016, 2007.

[8] L. Weiss and G. W. Schmid-Schonbein, "Biomechanical interactions of cancer cells with the microvasculature during metastasis", Cell Biochemistry and Biophysics, vol. 14, pp. 187-215, 1989.

\section{CONTACT}

* B. Lu, tel: +1-626-802-0898; lubo@mems.caltech.edu
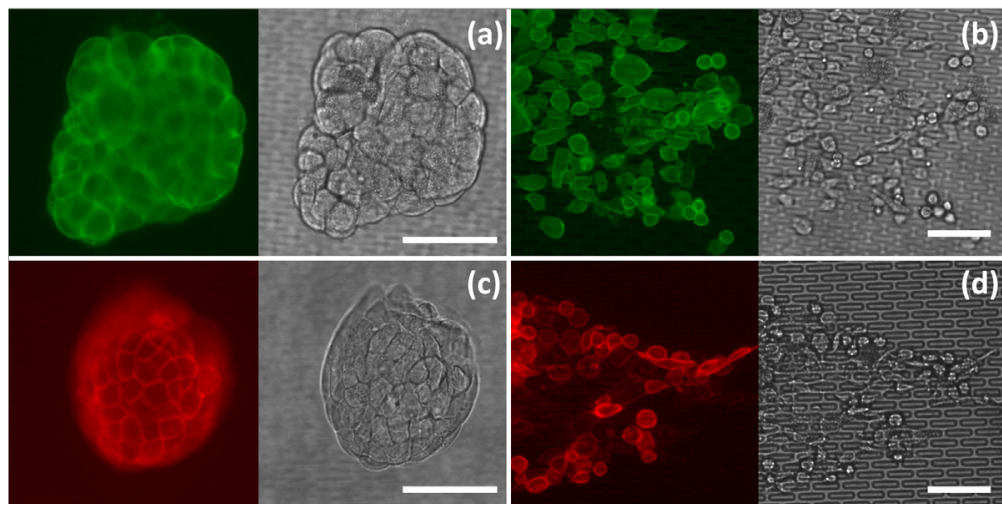

Figure 10: Immuno-staining of cultured $3 D$ tumor. (a) $3 D$ tumor stained with FITC-conjugated anti-EpCAM antibody. (c) $3 D$ tumor stained with PE-conjugated anti-human CD49b antibody. The $2 D$ on-filter (without parylene-HT coating) culture controls are shown in $(b) \&(d)$, respectively. (all scale bars: $100 \mu \mathrm{m}$ ) 